van der Kamp, B.J. and M. Spence. Stem diseases of lodgepole pine in British Columbia following juvenile spacing. 63(5): 334-339.

Van Wagner, C.E. See: Weber, M.G. et al.

Veilleux, J.-M.. See: Weetman, G.F. et al.

Weber, M.G., Monte Hummel and C.E. Van Wagner. Selected parameters of fire behaviour and Pinus banksiana Lamb. regeneration in eastern Ontario. 63(5): 340-346.

Weetman, G.F. Seven important determinants of Canadian silviculture. 83(6): 457-461.

Weetman, G.F., H.H. Krause, E. Koller and J.-M. Veilleux. 63(3): 184-192.

Wegwitz, E. See: Alfaro, I.R. et al.
Wellburn, G.V. Harvesting the future crop. 63(2): 103-106.

Wilford, D.J. A watershed perspective in a site specific world. 63(4): 246-249.

Willington, Robert P. Forestry herbicides and the aquatic environment. 63(4): 250-252.

Wood, J.E. and Richard Raper. Alternate strip clearcutting in upland black spruce. III. Regeneration options for leave strips. 63(6): 446-450.

Yeh, E.C.H. See: Cheliak, W.M. et al.

Zasada, John C., Rodney A. Novum, Christian E. Teutsch and Roseann Densmore. Survival and growth of planted black spruce, alder, aspen and willow after fire on black spruce/feather moss sites in interior Alaska. 63(2): 84-88.

\title{
Reviewers - The Forestry Chronicle, Volume 63, 1987
}

\begin{abstract}
My thanks are owing to the Associate Editors, without whom the Editor's job would be impossible. In addition, I wish to thank the following reviewers and apologize to those whose names I have missed.
\end{abstract}

The Editor

\author{
Léopold Anctil \\ Pierre Baril \\ Keith Belli \\ Réjean Bergeron \\ L. Bernier \\ Jean-Louis Boivin \\ D. Boylen \\ R.D. Briggs \\ H. Brix \\ R.G. Buchanan \\ Z. Chrosciewicz \\ M. Dessureault \\ M. Duinas \\ T. Easley \\ G.K. Edward
}

\author{
T.A. Erdle \\ A. Fuchs \\ Henry Gross \\ D. Haley \\ R. Hallet \\ W. Howard \\ M. Ker \\ H. Krause \\ N. Kreibery \\ D. Lavender \\ M.B. Lavigne \\ D. Lester \\ J.J. Lowe \\ M.K. Mahendrappa \\ J. Maini
}

P.L. Marshall

Anne Mauch

R.J. Miller

E. Needham

H. Ottens

F.L.C. Reed

H.T. Schreuder

P. Singh

N.J. Smith

T. Steele

D. Tait

A. Teskey

R. van den Dreissche

B. Wang

\section{Canadian Institute of Forestry}

\section{Institut Forestier du Canada}

\section{National Officers 1986-87 Bureau de direction national}

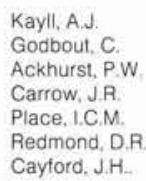

President 1 st Vice-President 2nd Vice-President Past President Editor

Production Manage Executive Director
Suite 1005, 151 Slater St. 992 Duchesneau 3950 Sunset Blvd.

Fac of Forestry, Univ of Toronto

3 Kitimat Cres.

643 Tillbury Ave.

Suite 1005,151 Slater St.

Ottawa, ON
Ste-Foy, PQ
N. Vancouver, BC
Toronto, ON
Nepean, ON
Ottawa, ON
Ottawa, ON

K1P $5 \mathrm{H} 3$

G1W 4 A9

V7R $3 \times 9$

M5S 1A1

$\mathrm{K} 2 \mathrm{H} 7 \mathrm{G} 4$

K2A $0 Z 9$

$\mathrm{K} 1 \mathrm{P} 5 \mathrm{H} 3$
Home

(613) $820-2805$ (418) $653-3080$

(519) 941.0792 (613) $829-2105$ (613) $728-8721$

\section{Section Officers 1986-87 / Responsables régionaux}

SECTION ALGONQUIN SECTION

Power. J.M. Chairperson, CIF

Yarranton, D. Director, CIF

Brand, D.G. Secretary, CIF

SECTION CARIBOO SECTION

Donnelly. Francis Chairperson, CIF

Donnelly, Francis Director, CIF

Robb, Darrell Secretary, CIF

SECTION CARIBOO CHILCOTIN SECTION

Dufresne, Keith Chairperson, CIF

Dufresne, Keith Director, CIF

Secretary CIF

SECTION CENTRAL ONTARIO SECTION

Smith, B.W. Chairperson, CIF

Graham, H.D. Director, CIF

MacNaughton, R.L Secretary, CIF

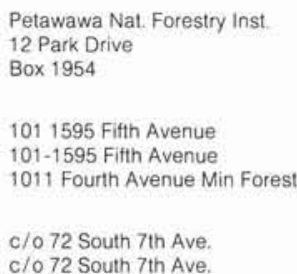

1184 Queen St E. 67 Amber Street
Chalk River, ON

Petawawa, ON

KOJ $1 \mathrm{JO}$

$\mathrm{K} 8 \mathrm{H} 2 \mathrm{E}$

KOJ $1 \mathrm{PO}$

Prince George, BC Prince George, BC Prince George, BC

V2L 3 L.

V2L. 3L9

V2L $3 \mathrm{H} 9$

Williams Lake, BC V2G 1 L3 Williams Lake, BC V2G 1Z5

Sault Ste. Marie, ON Sault Ste. Marie, ON Sault Ste. Marie. ON
P6A 6B4

P6A 2E6 P6A 6N6
Facsimile (819) 994-8697 (418) $643-2165$ 418) 643-799. (604) $387-1191$ (416) $978-5480$

(613) $234-2242$
(613) 687-4164

(613) $589-2880$ (613) $589-2880$ (613) $589-2880$

604) $561-1860$ (604) $561-1860$ (604) $964-3538$

604) $564-4115$ (604) $564-4115$ 604) $565-6100$
(613) $589-2275$ (613) $589-2275$ (613) $589-2275$ 